\title{
FROM GEOMETRIC TO COGNITIVE MAPS - A KEY ELEMENT FOR PERSONAL ROBOTS
}

\author{
Roland Siegwart, Shrihari Vasudevan, Adriana Tapus \\ Autonomous Systems Lab, Swiss Federal Institute of Technology Lausanne (EPFL) \\ CH-1015 Lausanne, Switzerland
}

Environment representation and situation awareness are key issue for personal and service robotics. Recent research in mobile robotics mainly concentrated on environment representations for efficient and robust navigation (e.g. Simultaneous Localization and Mapping). However, personal robots need competences that go beyond navigation and thus environment representations that include relationships and semantics of objects. Only this will enable personal robots to reason about the environment and develop cognitive competencies.

Many methods have been proposed to represent an environment in the framework of autonomous navigation, from precise geometric maps based on raw data or lines up to purely topological maps using symbolic descriptions. Each of these methods is optimal concerning some characteristics but can be very disappointing with respect to other requirements. However, none of them is able to cope with the large variety of environments that humans encounter in their daily live.

Most current approaches make a trade-off between precision and global distinctiveness. Precision and distinctiveness have a strong link with the level of abstraction of the features used for navigation (fig.1). Raw data represent the lowest level in the hierarchy of abstraction. Localization and mapping with raw data can result in high precision, but the required data volume scales very badly with the size of the environment, the distinctiveness of the individual data points is very low and semantic information cannot be integrated. An example of such an approach is Markov localization [Fox98, Thrun01]. The second level of abstraction 


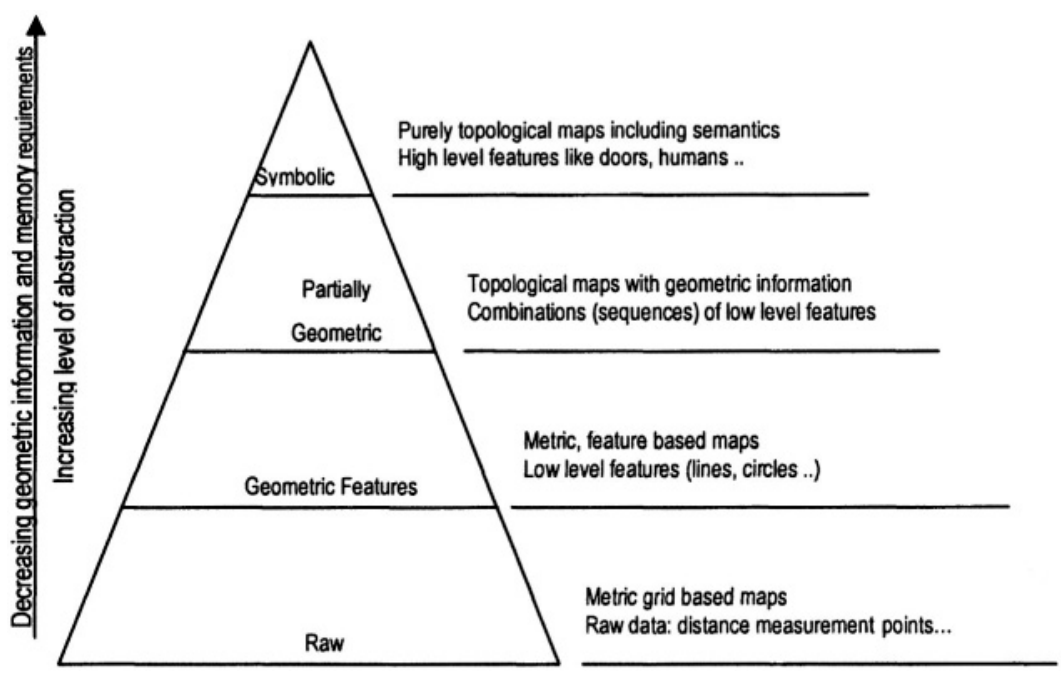

Figure 1: This figure depicts the hierarchy of abstraction levels towards cognitive maps. More we go up in the hierarchy, more we reduce the geometric information and we increase the distinctiveness and semantics information. For localization and mapping, geometric precision and distinctiveness is of importance, whereas for the development of cognitive competencies, semantics and relations between objects come forward.

corresponds to geometric features (lines, edges). The stochastic map technique to SLAM [Leonard92, Castellanos99, Dissanayake01] and the multi-hypothesis localization [Arras03] are typical examples belonging to this level. These approaches still feature high precision with reduced memory requirements, but have shortcomings concerning global distinctiveness and unmodeled events and are still purely geometrical. Partially geometric features correspond to the third level of the hierarchy. Representations using partially geometric features are for examples fingerprints (a sequence of low level features) described in [Lamon01, Lamon03], but also more bio-inspired approaches [Arleo00, Berthoz97, Hafner00] using neural networks, e.g. the work by Hafner [Hafner00] uses a neural network to create a topological map of the environment based on images of an omni-directional camera. On the highest abstraction level the environment is represented by a symbolic description. This can be very compact and distinctive, but reliable tools for extraction of high level features and appropriate concepts for probabilistic high-level knowledge representations are still under development. 


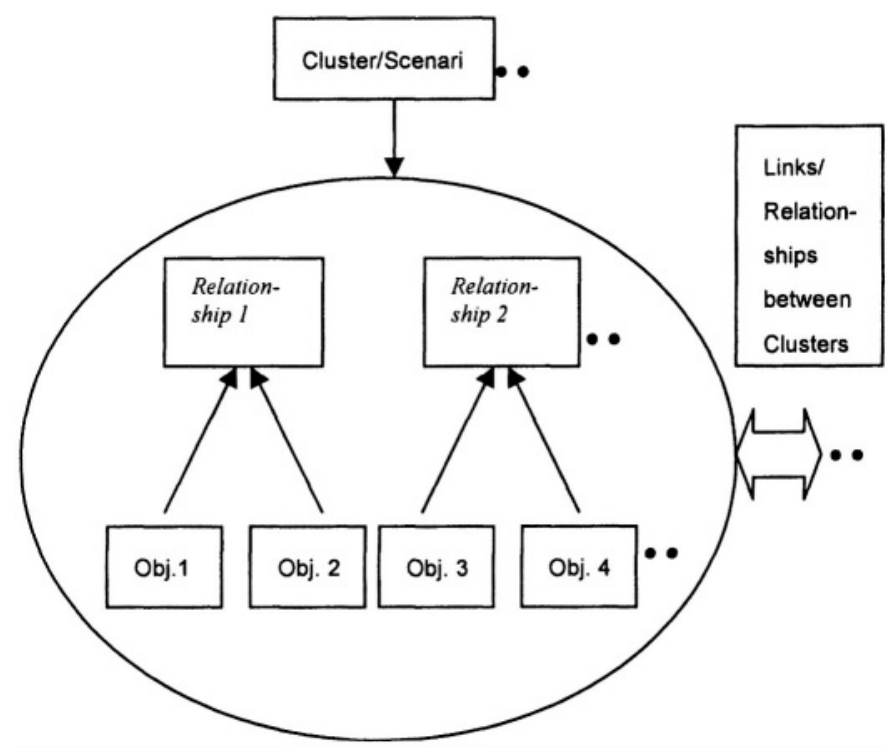

Figure 2: Cognitive Map: Relationship between objects - from relationships to scenarios

The aim of the present work is to suggest and discuss a multilevel representation approach for robust and compact environment representation with the goal of establishing cognitive maps for future service and personal robots. This is realized by integrating different level of abstraction and representations in a common framework for navigation and interaction. For example, to get from the airport to a given room in a hotel, maps of the city streets and of the hotel are necessary, but also knowledge about transportation systems and much more. Clearly, this task requires different representations and levels of abstraction for planning and decision-making. A crucial point is then the linkage between the different levels of abstraction so that the representation remains coherent over time. In the multi-level representation we propose, the mobile robot uses the most suitable level of abstraction, depending on the task it has to accomplish and the certainty about its pose. Moreover, depending on the actual situation, it has to be able to switch from one level to another (see figure 1).

In this paper we will discuss the possibility to integrate most recent results in mobile robot navigation in a multi-level hierarchical representation 
and we will present our first attempts towards multi-resolution, multirepresentation maps. The presentation starts with a general description of the proposed framework. We will then discuss typical sensors used in mobile robotics and their impact to the environment representation. Firstly our approach combining low-level features for local metric and fingerprints for global topological localization and mapping will be presented and highlighted with experimental results. Secondly our concepts towards cognitive representation are presented. The cognitive maps or mental maps term introduced for the first time in [Tolman48] - permit an encoding of the spatial relations between relevant locations or objects within the environment. More details with regards to the cognitive maps can be found in [O'Keefe78], The cognitive maps can be viewed as topological representations (see figure 2). The main idea is to combine objects and their relationships in a probabilistic object-graph model in order to enable planning and reasoning on an abstract level. Preliminary simulation results will outline this approach, its potential and related challenges.

\section{REFERENCES}

[Agrawal94] Rakesh Agrawal and H.V. Jagadish, "Algorithms for searching massive graphs," IEEE Transactions on Knowledge and Data Engineering, VOL 6, No 2, April 1994.

[Arleo00] Arleo A. and Gerstner W. (2000), "Spatial Cognition and Neuro-Mimetic Navigation: a model of Hippocampal Place Cell Activity", Biological Cybernetics, 83, 287-299

[Arras03] K.O.Arras, Castellanos, J.A., Schilt, M. and Siegwart, R., "Feature-based multihypothesis localization and tracking using geometric constraints," Robotics and Autonomous Systems, 1056(2003) 1-13, 2003

[Berthoz97] Berthoz A. (1997), "Le sens du mouvement", Editions Odile Jacob, Paris, 1997

[Castellanos99]Castellanos J.A., Tardos J.D., "Mobile Robot Localization and Map Building: A Multisensor Fusion Approach, ” Kluwer, 1999.

[Chaib-Draa98] Chaib-Draa, B. and Desharnais, J. "A Relational Model of Cognitive Maps “, International Journal of Human-Computer Studies, 49, 181-200, 1998

[Charniak91] Eugene Charniak, "Bayesian Networks without Tears," AI Magazine AAAI 1991

[Cohn02] Anthony Cohn, Aphrodite Galata, David Hogg, Derek Magee, Bernd Neumann, "Conceptual framework and knowledge representation tools for temporal and spatial context,” Technical Report, CSL IST - 2000 - 29375, 2002. 
[Crowley89] J. Crowley, "World modeling and position estimation for a mobile robot using ultrasonic ranging," Proc. of IEEE Int. Conf. on Robotics and Automation, pp. 674680, Scottsdale, AZ, May 14-19,1989.

[Dissanayake01] Dissanayake, Newman, Clark, Durrant-Whyte and Csorba, "A Solution to the Simultaneous Localization and Map Building (SLAM) problem”, IEEE Trans. On Robotics and Automation, Vol 17, No.3, June 2001

[Finn01] Finn V Jensen, "Bayesian Networks and Decision Graphs", Springer Verlag, 2001

[Fox98] Fox, D., "Markov Localization: A Probabilistic Framework for Mobile Robot Localization and Navigation," Ph.D. Thesis, Institute of Computer Science III. Bonn, Germany, University of Bonn, 1998

[Guo02] H. Guo and William H. Hsu. "A Survey of Algorithms for Real-Time Bayesian Network Inference," AAAI/KDD/UAI-2002 Joint Workshop on Real-Time Decision Support and Diagnosis Systems. Edmonton, 29 July 2002

[Hafner00] Hafner V.V., "Learning Places in Newly Explored Environments," in Meyer, Berthoz, Floreano, Roitblat and Wilson (Eds.), SAB2000 Proceedings Supplement Book, Publication of the International Society for Adaptive Behavior, Honolulu, 2000

[Lamon01] Lamon, P., I. Nourbakhsh, et al., "Deriving and Matching Image Fingerprint Sequencesfor Mobile Robot Localization," Proc. of the IEEE International Conference on Robotics and Automation (ICRA), Seoul, Korea, 2001

[Lamon03] Lamon, P., Tapus A., et al., "Environmental Modeling with Fingerprint Sequences for Topological Global Localization”, Proceedings of the International Conference on Intelligent Robot and Systems (IROS03) Las Vegas, USA, 2003.

[Leonard92] J.J. Leonard, H.F. Durrant-Whyte, "Directed Sonar Sensing for Mobile Robot Navigation, "Kluwer Academic Publishers, Dordrecht, 1992.

[Martinelli03a] Martinelli A and Siegwart R., "Estimating the Odometry Error of a Mobile Robot during Navigation," accepted at the European Conference on Mobile Robots (ECMR 2003), Warsaw, Poland, September 4-6, 2003

[Martinelli03b] Martinelli A, Tomatis N. et al, "Simultaneous Localization and Odometry Calibration", Proceedings of the International Conference on Intelligent Robot and Systems (IROS03) Las Vegas, USA, 2003.

[Martinelli03c] Martinelli A and Siegwart R., "Convergence of a Relative Map Filter for SLAM”, submitted at the ISRR03, Siena, Italy.

[O'Keefe78]O'Keefe, J. and Nadel, L. (1978). “The Hippocampus as a Cognitive Map”, Clarendon Press:Oxford.

[Pearl86] Judea Pearl, Fusion, "Propagation and structuring in beliefnetworks," Artificial Intelligence, Vol 29, Issue 3 (September 1986), pages 241 - 288

[Thrun01] S. Thrun, D. Fox, W, Burgard, and F. Dellaert, "Robust Monte Carlo Localization for Mobile Robots, ” In Artificial Intelligence (AI), 2001.

[Tolman48] Tolman, E. C. (1948), “Cognitive maps in rats and men”, Psychological Review, 55:189-208.

[Tomatis02] Tomatis N., Nourbakhsh I. and Siegwart R., "Hybrid Simultaneous Localization and Map Building: Closing the Loop with Multi-Hypotheses Tracking. " In Proceedings of the IEEE International Conference on Robotics and Automation, Washington DC, USA, May 11 - 15. 